\title{
ZERODUR stability in space environments
}

\section{A. Carré, T. Hull, A. Schüller, J. Krieg, T. Westerhoff}

A. Carré, T. Hull, A. Schüller, J. Krieg, T. Westerhoff, "ZERODUR stability in space environments," Proc. SPIE 11852, International Conference on Space Optics - ICSO 2020, 118524S (11 June 2021); doi: 10.1117/12.2599814 SPIE. Event: International Conference on Space Optics - ICSO 2021, 2021, Online 


\section{International Conference on Space Optics-ICSO 2020}

Virtual Conference

30 March-2 April 2021

Edited by Bruno Cugny, Zoran Sodnik, and Nikos Karafolas
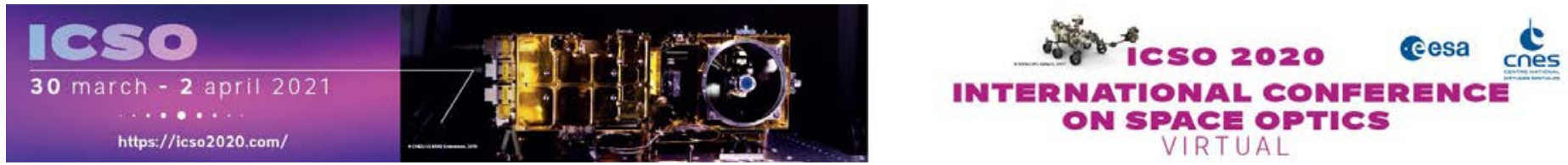

\section{ZERODUR stability in space environments}

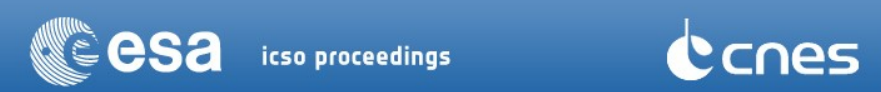

International Conference on Space Optics - ICSO 2020, edited by Bruno Cugny, Zoran Sodnik, Nikos Karafolas, Proc. of SPIE Vol. 11852, 118524S · @ 2021 ESA and CNES

CCC code: $0277-786 \mathrm{X} / 21 / \$ 21 \cdot$ doi: $10.1117 / 12.2599814$ 


\title{
ZERODUR $^{\circledR}$ stability in space environments
}

\author{
A. Carré*a, T. Hull ${ }^{\text {b }}$, A. Schüller ${ }^{\text {c }}$, J. Krieg ${ }^{\text {a }}$, T. Westerhoff ${ }^{\text {a }}$ \\ ${ }^{a}$ Schott AG Mainz, Hattenbergstraße 10, 55122 Mainz, DE; \\ bUniversity of New Mexico, 21 Algodon Lane Placitas, New Mexico 87043 USA; \\ c Physikalisch-Technische Bundesanstalt, Bundesallee 100,38116 Braunschweig, DE
}

\begin{abstract}
Recent comprehensive measurements of ZERODUR ${ }^{\circledR}$ 's response to realistic levels of ionizing radiation will be reported. This will compliment an update of ZERODUR ${ }^{\circledR}$ 's "history in space"[1] as implemented in both space agencies in Europe and in the United States. Specific recommendations for use of ZERODUR ${ }^{\circledR}$ in various orbit environments will be suggested.
\end{abstract}

Keywords: ionizing radiation, telescope, satellite, ageing, coefficient of thermal expansion, Zerodur ${ }^{\circledR}$

\section{INTRODUCTION}

Spaceborne reflective optics are usually made of materials having a very low thermal expansion coefficient around the targeted operating temperature of the space mission. The low dilatation property guarantees a form stability of the optic though all the thermal transients, however some other effects can contribute to the optic deformation such as compaction and or thermomechanical change induced by ionizing radiations. To date, the effect of the ionizing radiations on spaceborne optic material is not comprehensively understood, hence, for conservative reasons, the metrological error budgets associated to this phenomenon often overestimate the impact of radiations on the material. Studies carried out in the past where almost exclusively investigating the effect of a homogeneously distributed dose-deposition on some properties of optical material. Practically, the dose-deposition expected through the optical material varies strongly through the depth and depends on the type of mission considered (LEO/GEO, duration of the mission, local shielding of the optic due to the superstructure of the satellite, etc...). For all these reasons, the determination of a phenomenological ageing law describing the evolution of the characteristics of the material as a function of the local deposited ionizing dose is a very valuable tool in order to define a realistic error budget tailored to their specific mission parameters. In this present work, we investigated the influence of the electrons on the change of coefficient of thermal expansion of ZERODUR ${ }^{\circledR}$. In the first section we present the analytical approach we used in order to describe the impact of the ionizing radiations on the vertex radius of curvature of the optic. In the second section are presented the experimentation that we carried out in order to characterize the change in the thermal properties induced by the ionizing radiation. The third section describe the expected changes in the surface peak to valley and vertex radius of curvature under realistic electron irradiations.

\section{INDUCED CURVATURE. ANALYTICAL CALCULATION}

In this section, we present a textbook 1D analytical models based originally on the bimetallic assumption that we considered for the calculation of the irradiation induced curvature. This approach could appear obsolete compared to the powerful FEM solution routinely available nowadays; however, the typical dose-depth profiles that we are investigating in this work are difficult to address numerically: Typically, a decrease by several orders of magnitude of the deposited dose over micrometric scales are expected. These profiles would require a unusually tight FEM meshing of the optic in order to be comprehensively described at the macroscopic scale: such simulations could be achieved at the expense of exaggeratedly long simulation times. 


\subsection{Bimetallic approach}

Prior works investigating the effect of total ionizing irradiation on the change of curvature of optics were mostly based on the bimetallic approach. This model supposes that the optical device under consideration is composed of a homogeneously irradiated domain (front face of the optic) connected to a homogeneously shielded domain (back face of the optic). The geometrical determination of the irradiated domain and therefore of the estimated average irradiation level over this range were both arbitrary, these approximations biased the calculation of the curvature (and to the contrary, biased the estimation of the compaction law that had been derived using the bimetallic curvature expression). Because of this, we did not use directly the bimetallic description in this work.

\subsection{Continuous approach}

A continuous approach is described in Opto-structural Analysis: John W. Pepi [1]. The calculation of the Vertex Radius of Curvature (VRoC) of a rotation symmetric optic is given by:

$$
\frac{1}{R}=\frac{I}{\varepsilon_{e f f}}
$$

With

$$
I=\frac{d^{3}}{12}
$$

being the second moment of area, and $d$ the thickness of the optical element. And $\varepsilon(z)$ being the local strain defined:

$$
\varepsilon(z)=-\frac{1}{3} \frac{\left(\rho(z)-\rho_{0}\right)}{\rho_{0}}
$$

Where $\rho_{0}$ correspond to the local density of the sample taken at a reference temperature $T_{0}, z$ represents the position along the longitudinal axis of the cylindrical coordinate system, see Figure 1, and $\rho(z)$ is the local density taken at a temperature $T(z)$ with $\rho(z)=\rho(T(z))$.

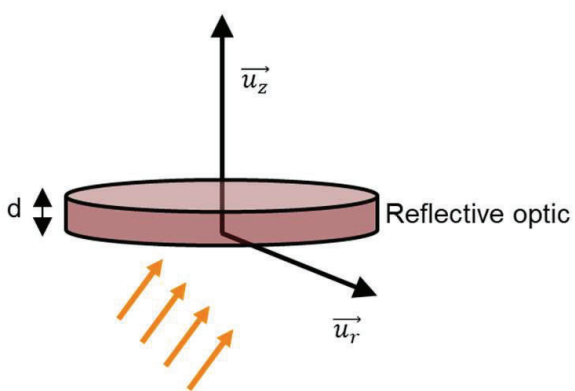

lonizing irradiation

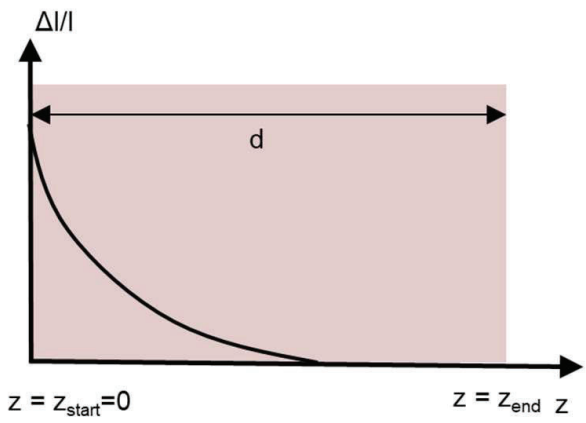

Figure 1: Left-Cylindrical coordinate system considered for the continuous approach. Right - sketch of a typical strain profile through the thickness.

Conventionally, we can use the center of the discs in order to perform the integration. However, if we subtract the average contribution of the strain to the strain profile function, we can then perform the integration using any possible coordinate origin along the sample thickness (this is especially useful if the origin of the coordinate system matches with the $\mathrm{z}=0$ origin of our dose-deposition profile). The averaged contribution of the strain is defined:

$$
\langle\varepsilon\rangle=\frac{1}{z_{\text {End }}-z_{\text {Start }}} \int_{z_{\text {Start }}}^{z_{\text {End }}} \varepsilon(z) \cdot d z
$$

Consequently, the effective integrated strain contribution can formally be defined: 


$$
\varepsilon_{e f f}=\int_{z \text { Start }}^{z_{\text {End }}}(\varepsilon(z)-\langle\varepsilon\rangle) \cdot z \cdot d z
$$

This continuous approach is extremely simple compared to e.g. the multi-metallic equation [3], in spite of its simplicity the results generated using this equation are very consistent with the multi-metallic approach and also with FEM results provided that radius/thickness ratio remains in the validity domain of the thin plate approximation.

\subsection{Strain model}

The local strain $\varepsilon$ over the depth $z$ of the optical device under consideration can be mathematically defined:

$$
\varepsilon(z)=-\frac{1}{3} \frac{\Delta \rho(D(z))}{\rho}+\operatorname{CTE}\left(T_{o p .} ; D(z)\right)\left(T(z)-T_{o p}\right)
$$

The first term on the right hand side corresponds to the radiation induced compaction, with $D(z)$ represents the total ionization dose (TID) depth profile and $\rho$ the local density of the material. This contribution is not discussed in the present paper as the validity of the presently available compaction models is still debated. This phenomenon will be further discussed in future work [4].

The second term on the right hand side of the aforementioned equation corresponds to the thermal strain induced by the change of Coefficient of Thermal Expansion (CTE) combined with the temperature gradient over the depth (remember that we are working presently with one-dimensional models).

Two scenarios will be discussed in the present paper, we will first investigate the change of sagitta induced by the homogeneous variation of the temperature close to the operating temperature of the optic, i.e. $T(z)=T \neq T_{o p}$, in this case the contribution $T(z)-T_{o p}$. is constant and the observed deformation are solely due to the local variation of the CTE induced by the ionizing radiation:

$$
\varepsilon(z) \propto \operatorname{CTE}\left(T_{o p} ; D(z)\right)
$$

Second, we will further complexity the model by superimposing a temperature field onto the CTE field:

$$
\varepsilon(z)=\operatorname{CTE}\left(T_{o p .} ; D(z)\right)\left(T(z)-T_{o p}\right)
$$

And we will check the form stability of different optical materials at different operating temperature.

\section{RADIATION INDUCED CTE VARIATIONS}

The estimation of the sagitta induced by spatial (depth) CTE variation presupposes the knowledge of the phenomenological law binding the local dose absorbed to the CTE variations. These results are only partially available in the literature, e.g. only for one given temperature or correspond to the temperature averaged CTE variation over a $T=0^{\circ} \mathrm{C}$ to $50^{\circ} \mathrm{C}$ range. Prior to our calculations, we had to quantify the impact of ionizing radiation on the thermal properties of Zerodur ${ }^{\circledR}$. In this section are presented the irradiation setup as well as the analytical CTE measurement performed on our coupons for the estimation of the dose-dependent CTE variations.

\subsection{Sample geometry}

Dilatometric measurements made in Schott AG metrological center typically require cylindrical samples $6 \mathrm{~mm}$ in diameter and $100 \mathrm{~mm}$ long. However the intended homogeneous irradiation with dose variation smaller than $10 \%$ of a $100 \mathrm{~mm}$ long sample in the radiation field of Gaussian shape could only be achieved at the expense of exaggeratedly low achievable dose rate. This would require a more time consuming and therefore costly irradiation run. In order to reduce the irradiation time we divided the samples in pairs of two $50 \mathrm{~mm}$ long rods that can be afterward stacked together into the dilatometer holder. With these smaller samples we could achieve the best compromise between radiation field homogeneity and dose rate. 


\subsection{Irradiation procedure}

The irradiation has been performed at the research linear accelerator of the Metrological Electron Accelerator Facility (MELAF) of the Physikalisch-Technische Bundesanstalt (PTB). The energy of the electrons used for the irradiation was $5 \mathrm{MeV}$. The electron beam is pulsed with a typical pulse width of $2.5 \mu \mathrm{s}$. The pulse repetition frequency used during the irradiation was $50 \mathrm{~Hz}$. An Integrating Current Transformer (ICT, Bergoz Instrumentation) is installed at the end of the accelerator beamline for non-destructive charge measurement of the electron pulses for beam monitoring.

At the end of the accelerator beam line the collimated electron beam passes through a $0.05 \mathrm{~mm}$ thick Ti foil vacuum window which scatters the beam. The diameter of this window is much larger than the beam width, thus all electrons detected by the ICT contributed to the radiation field. The scattering in the Ti foil results in a radial electron radiation field with a Gaussian lateral profile. The width $\sigma$ of the field increases with decreasing energy and increasing distance to the source. The width of the lateral dose distribution as function of the distance was determined experimentally by means of an ionization chamber matrix (PTW OCTAVIUS Detector 1500) as well by MC simulation (see Figure 2).

The samples were irradiated on the central axis of the radiation field at a distance of $1100 \mathrm{~mm}$. At this distance the width of the field is large enough ( $\sigma=92 \mathrm{~mm}$ for a $5 \mathrm{MeV}$ electron beam) to achieve a homogenous irradiation, i.e. a dose variation smaller than $10 \%$ over the surface to be irradiated (radius of $43 \mathrm{~mm}$ ). At this distance, the achievable mean dose rate amounts to about $10^{3} \mathrm{~Gy} \cdot \mathrm{min}^{-1}$.

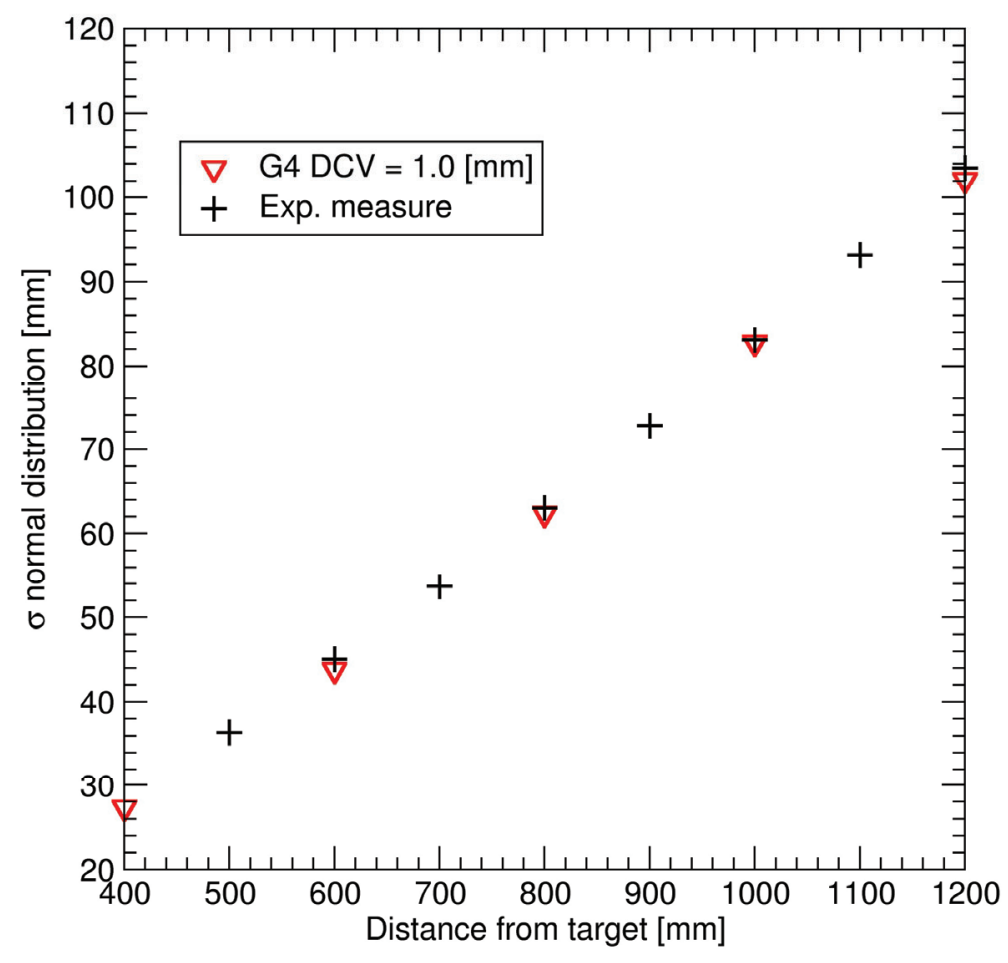

Figure 2: Cross: PTW Octavius measurement of the particle distribution for different DUT - source distances. Triangles: Geant4 estimated distribution for a default cut value of $1.0 \mathrm{~mm}$ (parameter defining the level of multiple scattering). The simulations are in good agreement with the experimental data.

The samples were irradiated with $10^{3}, 10^{4}, 10^{5}$, and $10^{6} \mathrm{~Gy}$, respectively. For the different deposited doses, the same setup and beam setting was used. Only the duration of irradiation was different. In order to achieve a homogeneous dose distribution also in the depth of the samples, they were irradiated from two sides. For this purpose, the samples were rotated after one half of the irradiation time. 


\subsection{Dose determination}

The absorbed dose to Zerodur ${ }^{\circledR}$ per incident electron in the current setup was determined by means of the Monte Carlo simulation Toolkit GEANT4 [5]. The number of electrons incident on the glass ceramic samples was measured in situ by means of a dedicated Faraday cup (FC) connected to a current integrator. The FC acting also as a sample holder (see Figure 3 ). Since accurate measurement of short intense charge pulses is challenging, the validity of this charge measurement was verified using a second independent method. For this purpose, the charge of the beam pulses in the beamline was measured inductively using a current transformer and the proportion that impinge on the cross-sectional area of the FC was determined.

The back side of the FC is a metal block, thick enough to completely stop high-energy electrons up to $10 \mathrm{MeV}$. Furthermore, it serves to dissipate the heat, in order to avoid a heat up of the samples during the irradiation. The front side of the FC is made of graphite to minimize electron backscattering from the metal block and loss of secondary electrons. In addition, the electrical and heat conductivity of this material avoids electrostatic charging as well as heat up of thesamples. The high-energy electrons pass through the graphite and the glass ceramic samples, depositing in it a part of their energy (dose) and are then stopped in the metal block at the backside.

The FC is electrically isolated from the ground by means of a wood wedge. The leakage current was negligible, i.e. all electrons collected by the FC are counted by the current integrator (Ortec 439). A resistor-capacitor circuit (120 Ohm, $15 \mu \mathrm{F}$ ) was mounted at the input of the current integrator to keep the current from the short high charge pulses low enough.
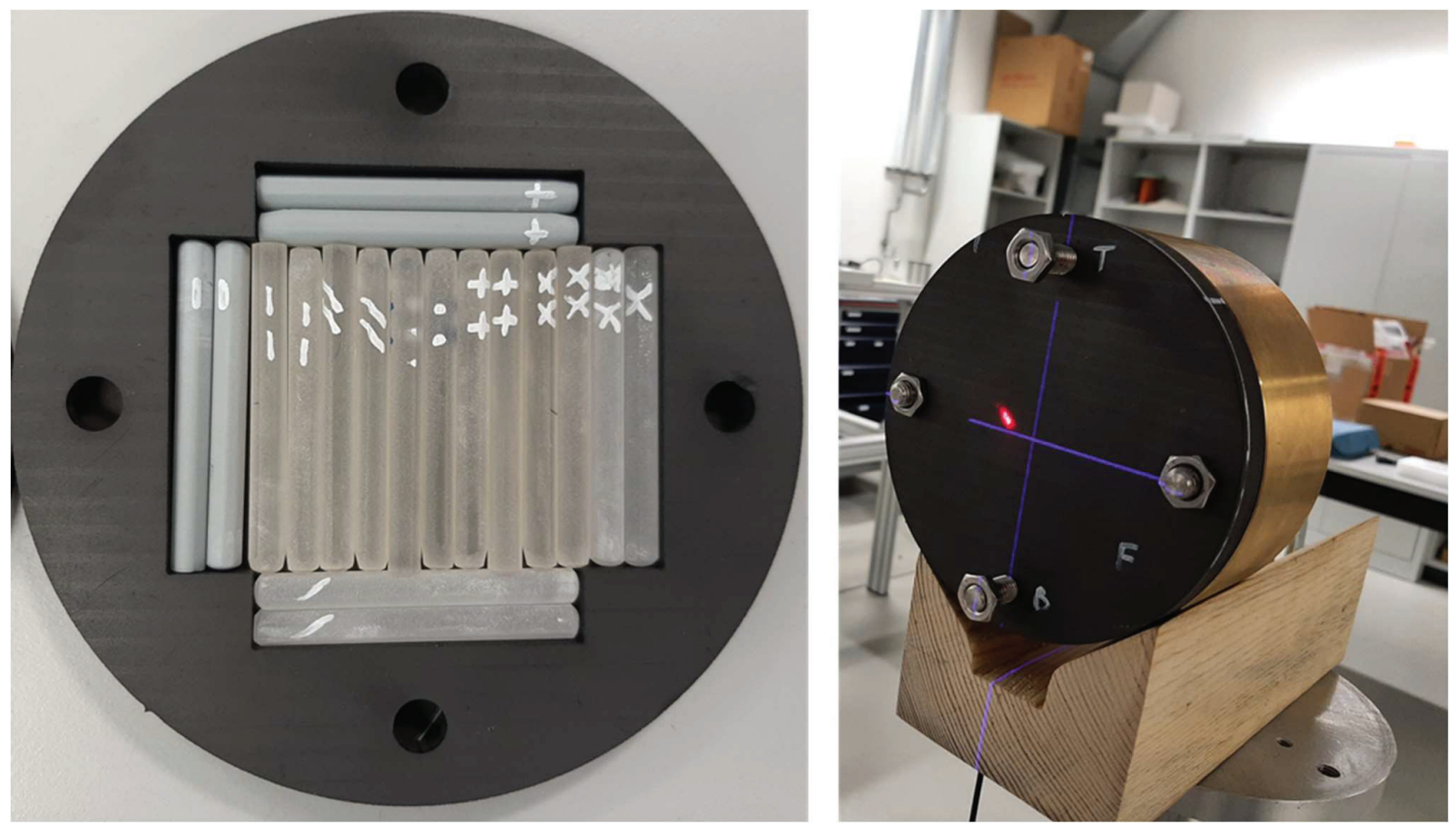

Figure 3: Left hollow in the graphite FC where the pair of $2 x 50 x 6 \mathrm{~mm}$ samples have been placed. Right the FC prior to an irradiation test, the samples are hold between a graphite front plate and a graphite + bronze background. The whole FC is connected to a current integrator (connection not visible on this picture). The FC is electrically isolated from the ground by means of a wood wedge. The electrical isolation ensure that leakage current is negligible so that all collected electrons are counted by the current integrator. The blue laser lines indicates the central beam axis of the radial field distribution. The red laser spot indicates the point where the temperature is monitored by the pyrometer.

This in situ measurement setup is especially valuable for long time irradiation run were the intensity of the electron beam could drift or if some other disruptions would occur during the irradiation. 
To verify the charge measurement method the charge of the beam pulses in the beamline was measured simultaneously using FC and by means of the ICT. The fraction $f$ of electrons that impinge on the cross-sectional area of the FC is a function of the FC radius $R$, the distance $z$, and the width $\sigma$ of the Gaussian shaped field:

$$
f=1-e^{-\frac{R^{2}}{2 \sigma(z)^{2}}}
$$

The measured fraction of electrons collected by the FC agrees well with the theoretical value $f$ (see Figure 4). ICT and FC give the same result when the $\mathrm{FC}$ is directly in front of the exit window, i.e. $f(\mathrm{z}=0)=1$. It therefore can be concluded that the charge measurement methods by means of FC and ICT agree $\left(\mathrm{QFC}_{\mathrm{FC}}(\mathrm{z}=1100 \mathrm{~mm})=f(\mathrm{z}=1100 \mathrm{~mm}) \times \mathrm{Q}_{\mathrm{ICT}}\right)$.

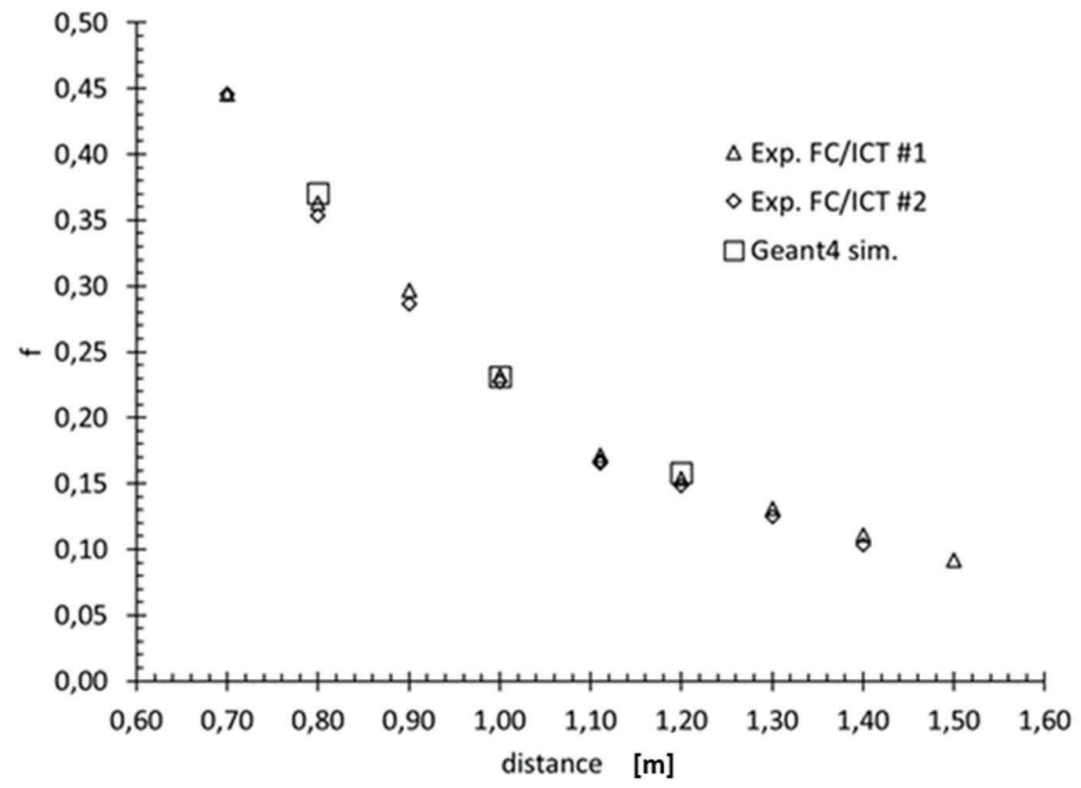

Figure 4: The fraction of electrons collected on the FC. Triangles: ratio between the measured charge from the FC and the charge measured by the ICT. Diamonds: $f(z, \sigma)$ from measured width $\sigma$. The simulated results (squares) are in good agreement with the experiments.

The estimation of the deposited dose has also been achieved using Geant4. Practically simulations have been performed using a fixed number of electrons being directly fired onto the target $N_{\text {Sim. }}$. and the corresponding simulated dose-depth within ZERODUR ${ }^{\circledR}$ has been determined $\left(D_{\text {Sim. }}\right)$. Using these two numerical values, we could determine the amount of electrons $N$ required in order to experimentally achieve an average target TID of $\langle D\rangle$ in our samples:

$$
\langle D\rangle=\frac{1}{V} \int D \cdot d V=\frac{N}{N_{\text {Sim }}} \times \frac{1}{V} \int D_{\text {Sim. }} \cdot d V
$$

In this above formula $V$ represents the volume of a CTE sample. We can reformulate this equation into:

To this amount of electrons $N$ corresponds a charge $q$ defined:

$$
N=\frac{N_{\text {Sim. }}\langle D\rangle}{\frac{1}{V} \int D_{\text {Sim. }} \cdot d V}
$$

$$
q=N * C
$$

Where $C$ represents the elementary charge of an electron. The charge $q$ is the one that must be collected on the Faraday cup in order to achieve the target average dose $\langle D\rangle$.

The irradiation at a given target average TID has been systematically performed in two phases. During the first phase we irradiated the samples until we reached one half of the target charge on the Faraday cup, then we flipped the samples (front to back) and the irradiation run were then finished, i.e. till we reach the total targeted charge on the FC. The corresponding dose depth profile is exemplary pictured for a TID of $10^{5}$ Gy on Figure 5. 


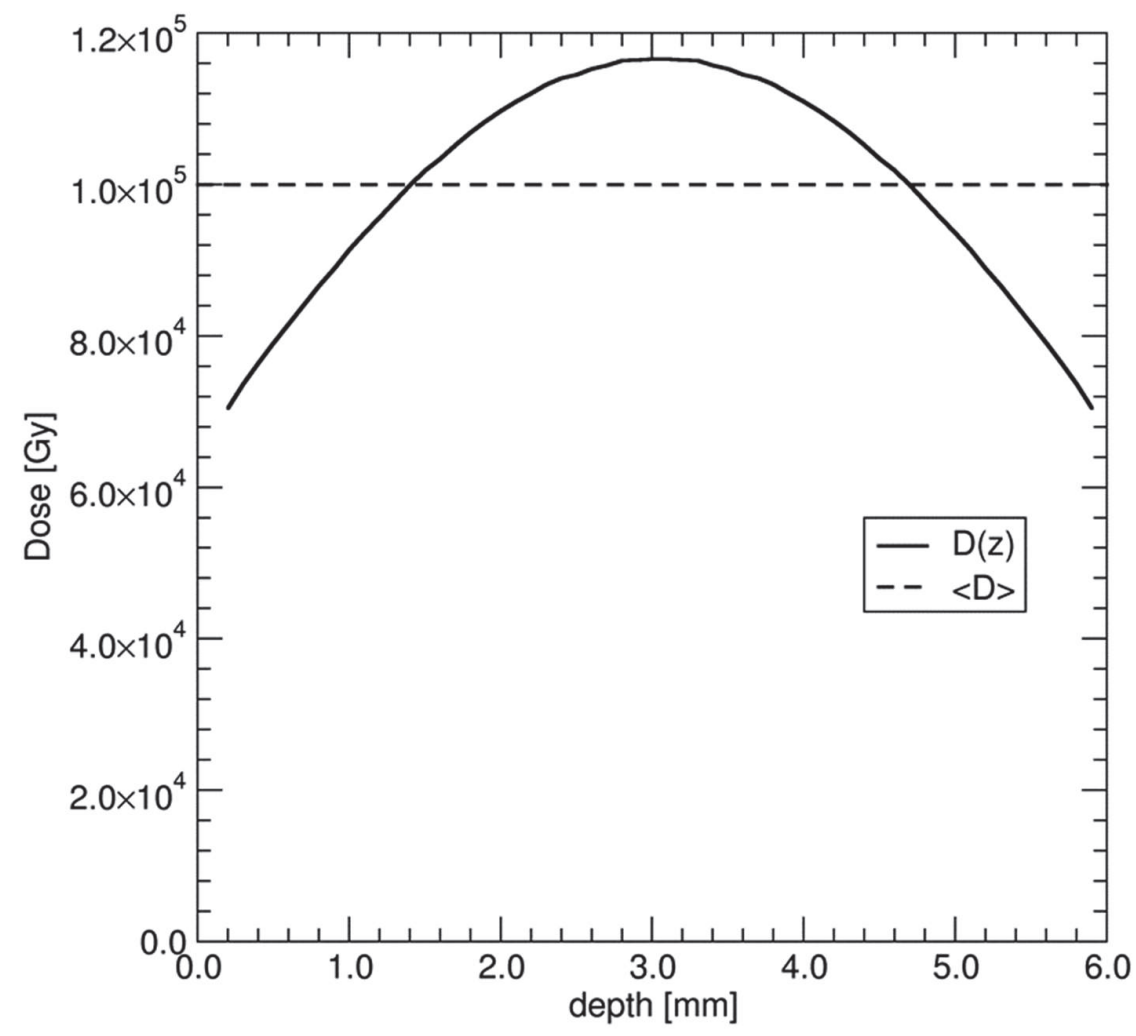

Figure 5: Continuous line: dose depth profile within ZERODUR® samples simulated for a target TID of $10^{5}$ Gy. Dashed line: Averaged TID value $<D>$ over the depth.

\subsection{Temperature monitoring}

The heat up (as a corollary of the dose deposition rate) within the sample holder should be minimized. From previous Electron Spin Resonance investigations on glass ceramics and also from photoluminescence measurements is known that some defect healing could be thermally activated [6].Thus, by keeping the temperature close to the room temperature we avoid potential temperature activated effects at higher temperature. A pyrometer was used for in situ monitoring of the temperature of the sample holder.

The defects induced by the ionizing radiation in the samples may also partially reduced by thermally induced relaxation and healing during the time span between the irradiation and the CTE measurement. In order to freeze the damaged state of the samples, we conserved them at low temperature $\left(-17^{\circ} \mathrm{C}\right)$ directly after irradiation. The samples have been transported from PTB to the Schott Marienborn metrological center using a dedicated container that kept the samples at low temperature $\left(\leq-17^{\circ} \mathrm{C}\right)$, and once in Marienborn, the samples have also been kept by $\leq-17^{\circ} \mathrm{C}$ prior to analyses.

\subsection{Analytical measure}

As with most mirror materials, ZERODUR ${ }^{\circledR}$ averaged CTE value over the $\mathrm{T}=[0 ; 50]{ }^{\circ} \mathrm{C}$ temperature range is expressed, so-called CTE( $0 ; 50)$. This figure or merit is not fully relevant for those designing and operating space optics. Frequently, the embedded mirrors are designed to be optimal around a given operating temperature, for example the $[0 ; 20]{ }^{\circ} \mathrm{C}$ range. It is important to determine the instant CTE value at each temperature as well as the change induced by the radiation on the mirror in order to characterize with precision the surface level effect of local temperature variation around the operating temperature. 
The instant CTE, CTE(T), corresponds to the first derivative of the Total Change of Length (TCL $=\Delta l / l=\left(l(T)-l_{0}\right) / l_{0}$ with $l_{0}$ the length of the samples at a given constant temperature $T_{0}$ ) with respect to the temperature $T$.

$$
\operatorname{CTE}(T)=\left.\frac{d(\Delta l / l)}{d T}\right|_{T}
$$

In this work, the TCL measurements have been achieved by means of an optical dilatometer, the measurements are performed dynamically over a $[-30 ; 30]^{\circ} \mathrm{C}$ temperature range, see Figure 6 .

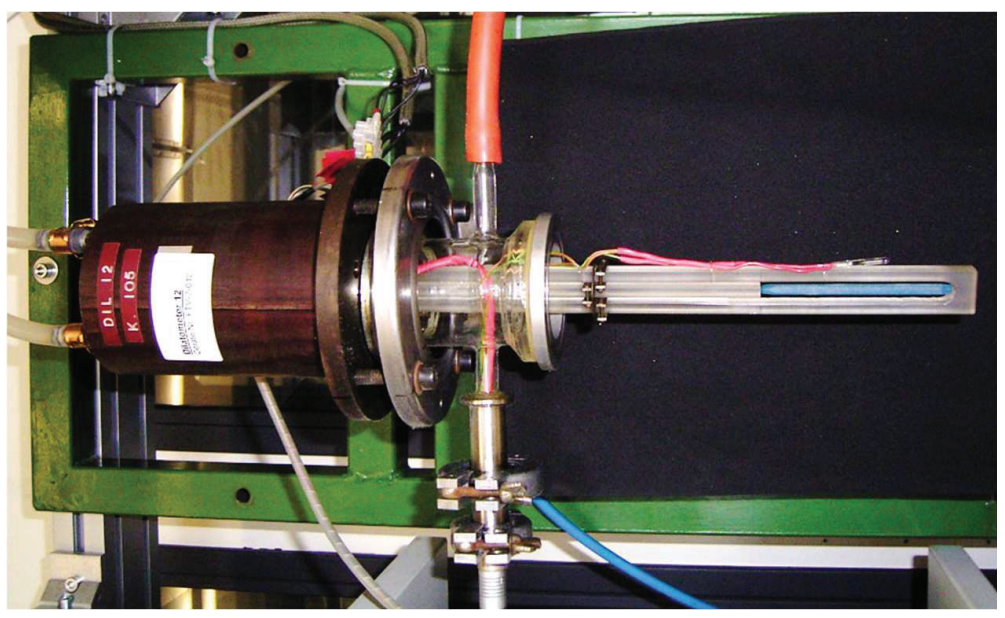

Figure 6: Picture of the optical dilatometer used for the estimation of the CTE. For our measures the irradiated rods have been pairwise stacked in place of the blue rods.

Due to the smallness of the changes observed, several iterations were required in order to resolve properly the CTE values. To this end we used different measurement protocols based different time-temperature cycles in order to determine the best accuracy the CTE. For the determination of the measurement protocols, we systematically used different pair of ZERODUR ${ }^{\circledR}$ rods in order to perform CTE characterization on as irradiated samples. An exemplary $t$ - $T$ program is picture on Figure 7.

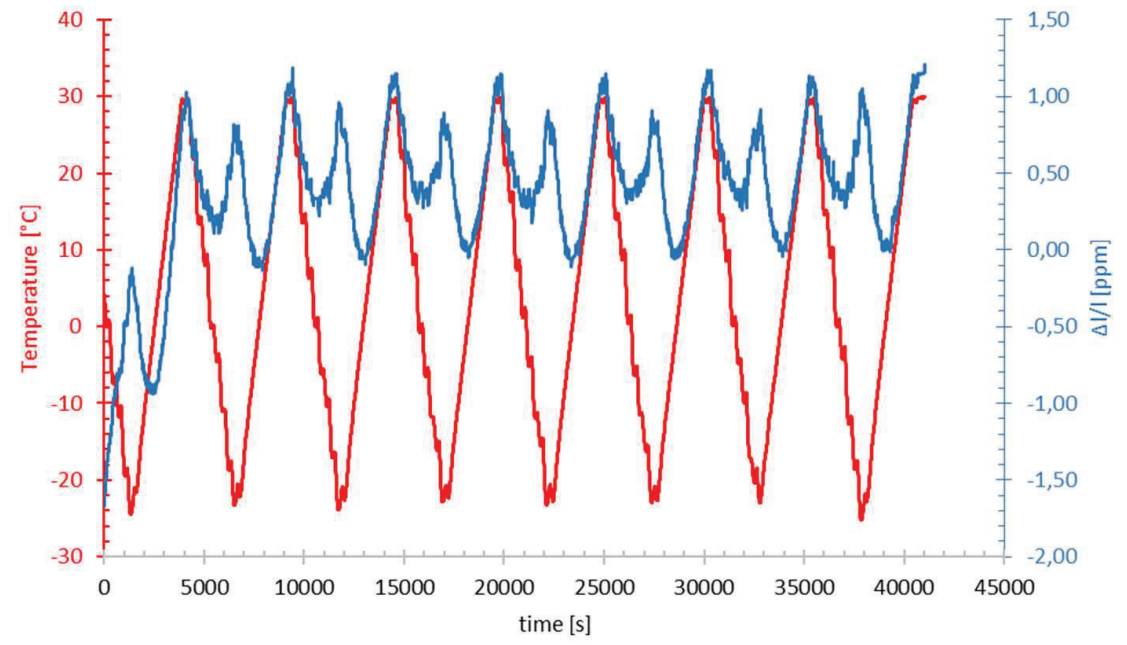

Figure 7: Dilatometric measurement curves. In red the time-temperature program, in blue the total change of length $\Delta \mathrm{l} / \mathrm{l}$ as a function of time. The temperature dependence of the TCL can derived by plotting the $\Delta l / l$ as a function of temperature. 
Previous work made in this field by (Paige L. Higby [6]) suggested that the TCL measurement could eventually heal defect the samples especially during the TCL measurement over the higher temperature (at that time $300 \mathrm{~K}$ ). Being aware of this difficulty, we purposely performed some of our first TCL characterization starting from a low temperature plateau $-10^{\circ} \mathrm{C}$ followed by a further decrease of the temperature down to $-20^{\circ} \mathrm{C}$ (at $-1.2 \mathrm{~K} \cdot \mathrm{min}^{-1}$ ). This phase was then followed by an increase up to $10^{\circ} \mathrm{C}\left(\right.$ at $\left.+1.2 \mathrm{~K} \cdot \mathrm{min}^{-1}\right)$ then again followed by cooling down to $-20^{\circ} \mathrm{C}\left(\right.$ at $\left.+1.2 \mathrm{~K} \cdot \mathrm{min}^{-1}\right)$. We repeated this program with increasing progressively the upper temperature bound. This procedure has been designed in order to check whether any healing effect could be observed (we would then observe a systematic drift of the CTE over the $T$ - $t$ iteration). Interestingly, in spite of having cautiously investigated any thermal relaxation threshold, we could not observe any healing effect similar to those reported by P.L. Higby in her pioneering work. The thermal activated healing process having been set aside we performed the standard TCL investigation using the time-temperature program described in Figure 7.

\subsection{CTE variation law}

The different TCL curves have been fitted over a $[-20 ; 30]^{\circ} \mathrm{C}$ by means of a degree 5 polynomial. These polynomials have been thereafter derived with respected to the temperature and the local derivative of the TCL, i.e. the CTE:

$$
\operatorname{TCL}(T) \approx \operatorname{TCL}\left(T=T_{0}\right)+\left.\left(T-T_{0}\right) \frac{\partial T C L(T)}{\partial T}\right|_{T=T_{0}}=\operatorname{TCL}\left(T=T_{0}\right)+\left(T-T_{0}\right) * \operatorname{CTE}\left(T=T_{0}\right)
$$

The CTEs values calculated for different averaged deposited doses have been graphically presented on Figure 8 below:

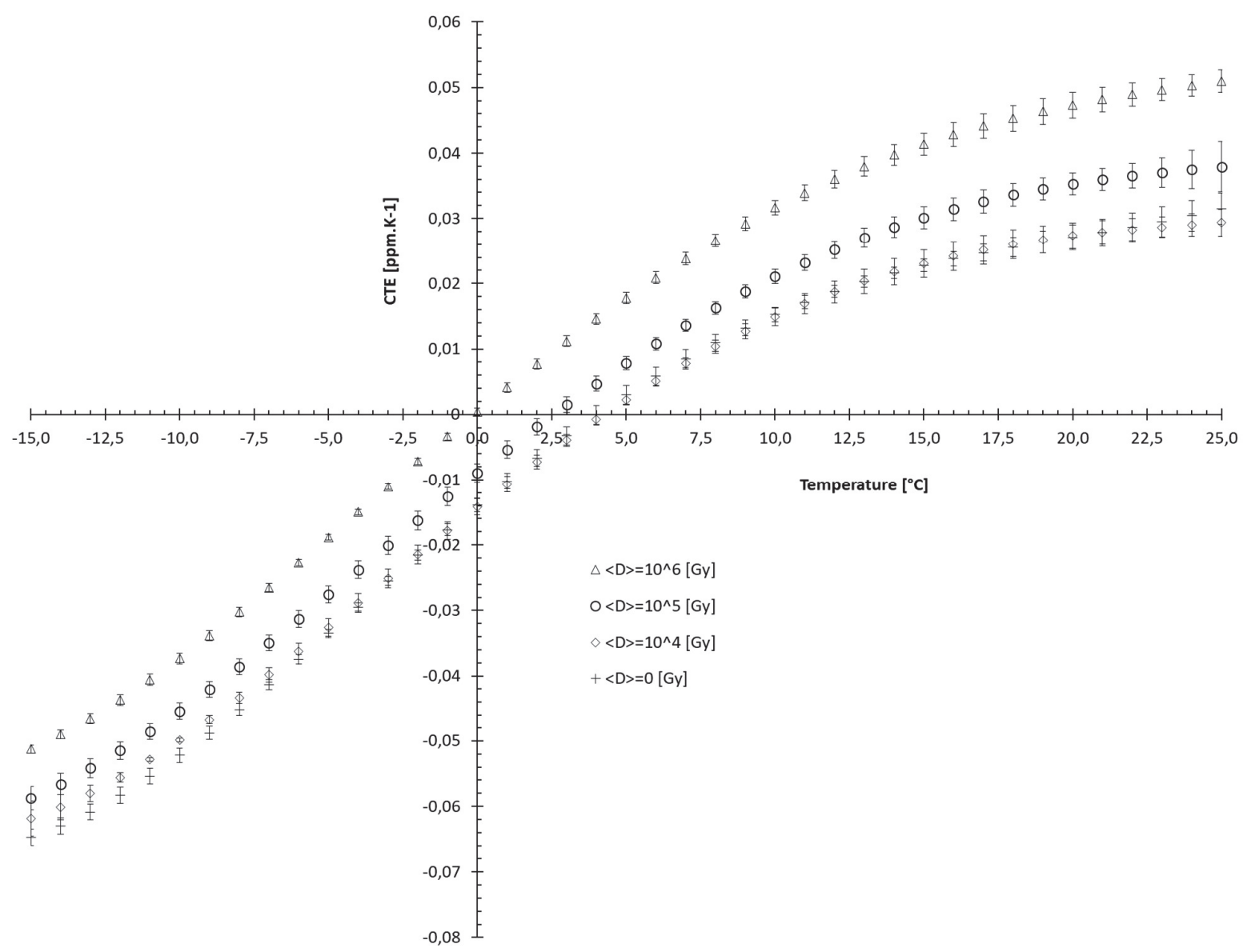

Figure 8: Cross- CTE determined for the samples prior to irradiation (Standard ZERODUR®). Triangle, circle and squared symbols : CTE values determined respectively for $10^{6}, 10^{5}$ and $10^{4}$ averaged TID $\left(\langle D>)\right.$, the CTE observed for the $10^{3}$ Gy case were not significantly different from the CTE values determined for $10^{4} \mathrm{~Gy}$, consequently we have not included these data on the graph. The CTE 
values corresponding to the unirradiated coupons are represented with crosses. The confidence interval has been calculated with $\alpha=0.05$.

Using these data, we proposed a power law estimation of the local CTE variation as a function of the TID, $\triangle C T E\left(T_{o p .} ; D\right)=A_{T_{\mathrm{op}}} \times D^{B T_{o p}}$. Following this formalism, the overall CTE can then be written:

$$
\operatorname{CTE}\left(\mathrm{T}_{\text {op. }} ; \mathrm{D}\right)=\operatorname{CTE}\left(\mathrm{T}_{\mathrm{op}}\right)+A_{T_{\text {op }} .} \times D^{B_{T o p}} \text {. }
$$

The power law has already successfully be used in order to characterize compaction [7][8]. Moreover, this formulation naturally takes into account the fact that for zero dose, the induced changes must also be zero.

The offset between the $10^{6} \mathrm{~Gy}$ and $10^{5} \mathrm{~Gy}$ instant CTE change appears to be linearly temperature dependent, in order to quantify this visual impression we plotted the difference between these two set of CTE data:

$$
\operatorname{CTE}\left(\mathrm{T}_{\mathrm{op} .} ; 10 \times \mathrm{D}\right)-\operatorname{CTE}\left(\mathrm{T}_{\mathrm{op} .} ; \mathrm{D}\right)=A_{T_{o p .}} \times\left[(10 \times \mathrm{D})^{B_{\text {Top. }}}-D^{B_{\text {Top. }}}\right]
$$

This above relation has been plotted on Figure 9:

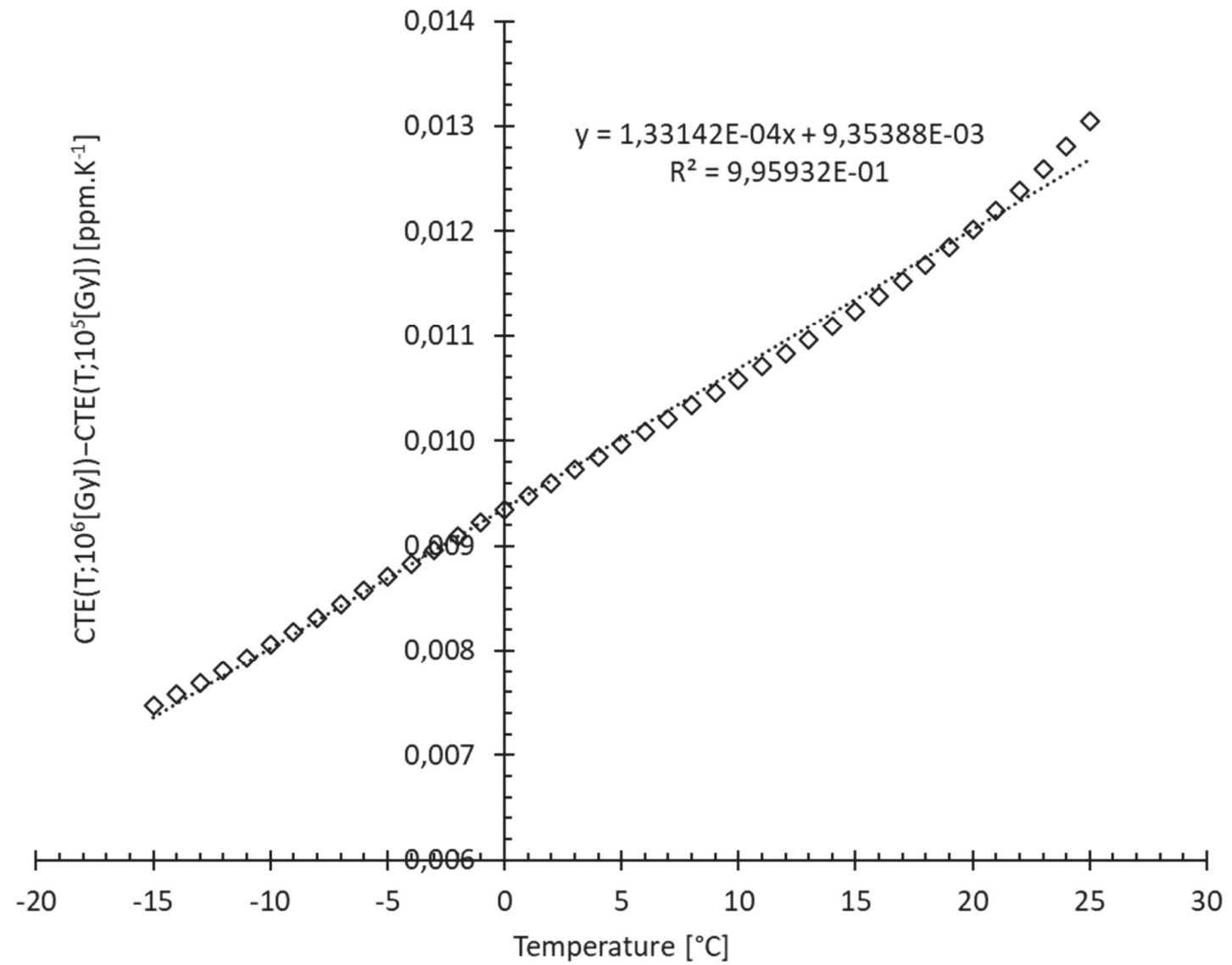

Figure 9: The only contribution of irradiation to the instant CTE change have been quantified for the $10^{6}$ and $10^{5}$ Gy data. The relation appears to be strongly linear with the temperature.

The differences between the CTE for $10^{6}$ and $10^{5}$ Gy confirmed to be reasonably well described by a linear regression law. Based on this observation we arbitrarily decided to pack all the temperature dependency into the prefactor $A_{T_{o p}}$ and to set the exponent $B_{T_{o p .}}=B$ as being independent from the temperature. Numerically we estimated that the prefactor $B$ is quite close to 0.5 and we decided for the convenience of the numerical implementation to fix it equal to 0.5. Following this ansatz the CTE change law expressed in $\mathrm{ppm} . \mathrm{K}^{-1}$ as a function of the temperature in ${ }^{\circ} \mathrm{C}$ and the deposited dose expressed in Gy can then be formally written:

$$
\operatorname{CTE}(T ; \mathrm{D})=\operatorname{CTE}(\mathrm{T})+\left(1.5096 \times 10^{-5}+T \times 2,1487 \times 10^{-7}\right) D^{0.5}
$$


As the variations of Zerodur ${ }^{\circledR}$ CTE are by far smaller than initially assumed, only the data determined for an average deposited dose of $10^{6}$ and $10^{5}$ Gy could be used for the derivation of a phenomenological law. The authors are aware of the sparsity of these data, however with this prospective work we are now able to propose further irradiation points, e.g. $2 \times 10^{5} \mathrm{~Gy}$, in order to consolidate this phenomenological description.

\section{SIMULATIONS}

\subsection{FEM validation of the $1 \mathrm{D}$ analytical model}

The continuous 1D description of the surface curvature is valid as long as we are working in the realm of the thin plate approximation, i.e. some drifts could be expected between the FEM measurement and the 1D analytical model if the diameter/thickness ratio is not large enough (typically $\geq 10: 1$ ). An aspect ratio of 10:1 is typical of many open-back lightweight space borne mirrors up to $1.5 \mathrm{~m}$ diameter delivering $200 \mathrm{~Hz}$ free-free first eigenfrequency. Larger lightweight mirrors may go to somewhat chunkier aspect ratios. As a preamble to our analyses, we wanted to cross-check the agreement between the 1D plate model and the FEM predictions. To this end, we used the FEM simulations model that we considered for defining the DOE of a ZERODUR ${ }^{\circledR}$ compaction law, see [4]. The meshing of the structure has been made by means of ANSYS and the calculation has been made with ABAQUS on a axial symmetric system with only one fixed point. The finite element size in the z-direction were adapted to the discretization step of the dose-depth, i.e. 0,05 mm, whereas their radial extension was about ten times this value. The samples under consideration are assumed to be irradiated with a 0,5 $\mathrm{MeV}$ and 1,5 MeV e- flux. Due to the different e- penetration depth at these energies we considered two different geometries. Coupons having a $2 \mathrm{~mm}$ thickness are irradiated with $0,5 \mathrm{MeV}$ whereas coupons having a $5 \mathrm{~mm}$ thickness are irradiated with 1,5 MeV. The dose-depth profiles along the z-axis (assuming a polar representation of the coordinate system) are translated into a strain profile by means of a compaction law. The final change of curvature of the optic under consideration is then calculated by relaxing the stress field within the material.

These FEM data have then been compared to the analytical results generated by means of the 1D bending approach. The numerical calculations have been achieved by means of a Python script based on the SciPy library. We used the same sample geometry as those considered for the FEM for the calculation of the VRoC (solely determined by the relaxation of the irradiation induced strain profile along the length of the optic as well as for the Peak-to Valley (PV) surface change VRoC that requires the VRoC as well as the radius of the optic for the calculation of the SAG). The numerical agreement is excellent see Figure 10.

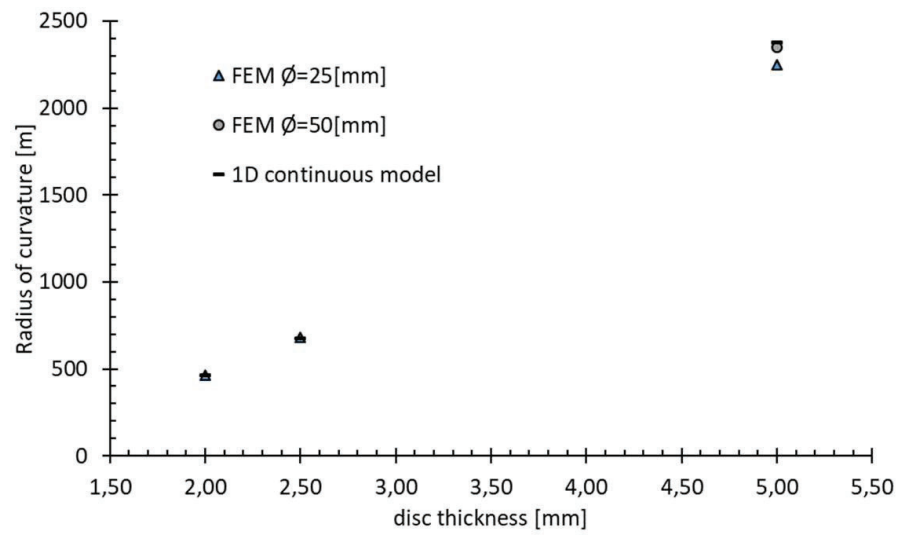

Figure 10: Calculation of the radius of curvature (VRoC) for three different disc thickness, namely 2,0;2,5;5,0 $\mathrm{mm}$ for a e-E=0,5 MeV dose-depth profile, peak dose is equal to $10^{4} \mathrm{~Gy}$ in this case. The (-) corresponds to the results generated using the gradient equation, the triangle and the circle to the results generated using Abaqus on two different disc radius. We can see that the larger is the radius of the disc the closer the FEM results are converging to the analytical solution. 


\subsection{Dose-depth profile over a typical spaceborne mirror}

Dose-depth profile are frequently formulated in terms of dose deposited by an omnidirectional particle flux at the center of a solid sphere as a function of the sphere radius, see Figure 11 and [9]. The ionizing dose deposition calculated in this way is overestimating the dose deposition that one would expect on a real embedded mirror. In a satellite system, the ZERODUR ${ }^{\circledR}$ mirror would be shielded from the omnidirectional flux by a tube assembly consisting of the outer stray light baffle, the telescope's metering structure plus thermal shields. In order to estimate the shielding influence of the telescope tube assembly onto the primary mirror, we designated a reference tube assembly $2010 \mathrm{~mm}$ in length of aluminum (2.54 $\mathrm{mm}$ thick). In actual telescopes, the effective shielding thickness typically includes substantially more shielding mass than our reference case. The primary mirror assumes a $10 \mathrm{~mm}$ thick ZERODUR ${ }^{\circledR}$ facesheet on the front of support pocketed to lightweight. For simplicity, we neglected shielding from the secondary mirror assembly (see Figure 12). Moreover, since there was miniscule penetration of radiation beneath the facesheet, we legitimately neglected the dose-depth profile within the isogrid structure of the telescope. It is assumed that there will be a strong back, instruments and a spacecraft aft of the primary mirror; thus, irradiation coming from the back will be negligible. In this work. The exclusive effect of e-.for the characterization of the CTE variation laws has been considered. Consequently, in those subsequent sections, we only focus on the contribution of e- on the optic deformation.

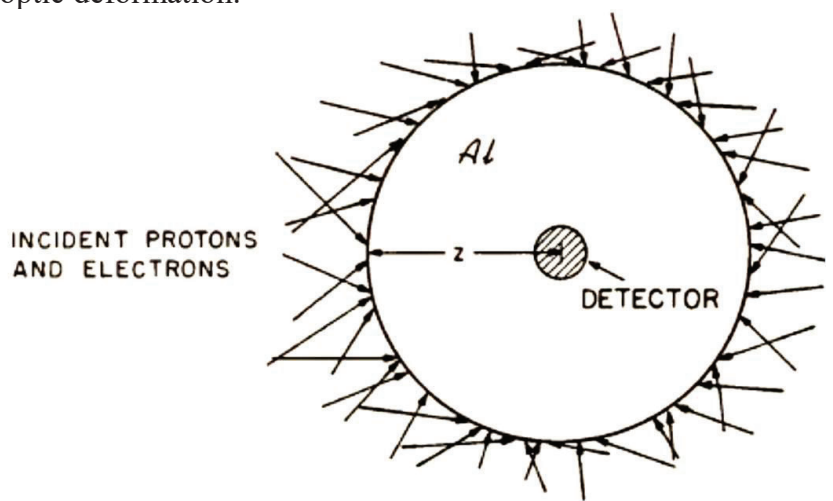

Figure 11: Detector located at the center of a Al sphere radius as described in the documentation manual of the Shieldose2 software. 


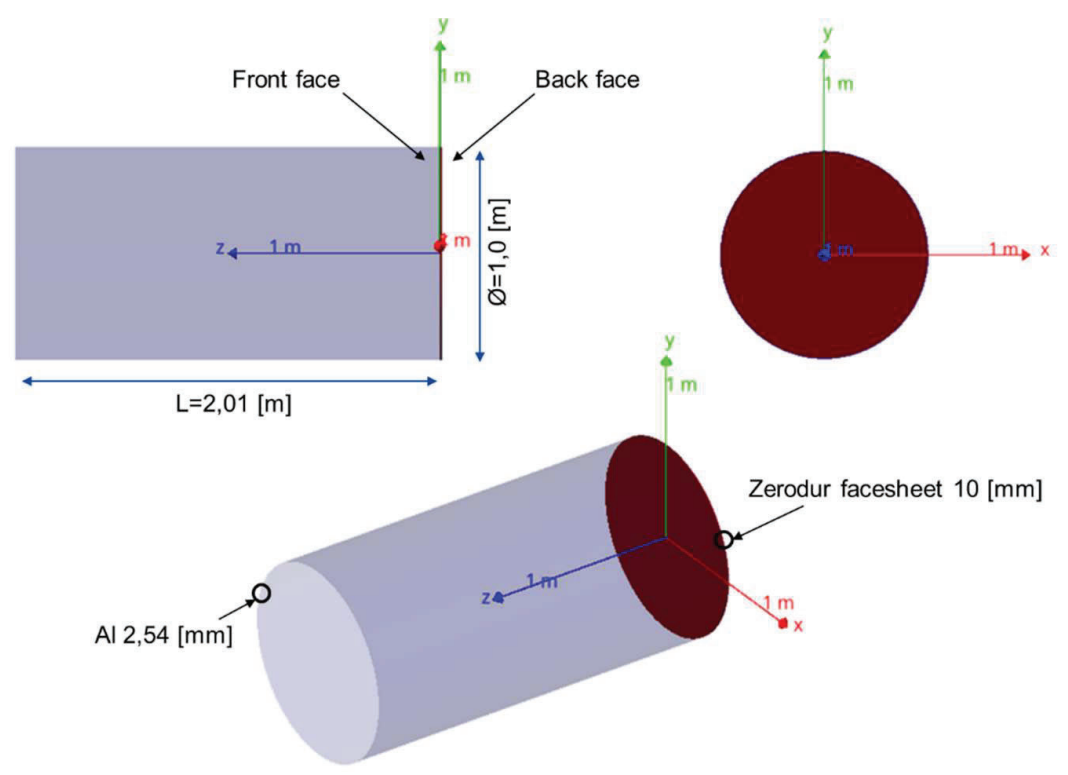

Figure 12: Simplified model of a telescope. The facesheet of the telescope (burgundy) is partially shielded from the omnidirectionnal e-flux by a 2,54 $\mathrm{mm}$ of Al allocated to the tubular baffle and outer thermal shell. This tube providing shielding is 2,01 $\mathrm{m}$ long and the radius of the optic is 1,0 $\mathrm{m}$ in diameter. We only considered the front sheet of the telescope and not the isogrid structure behind nor the whole satellite in order to further simplify our dose deposition model.

The particle flux distribution for a given orbit can be calculated by means of Spenvis [10]. The particle trajectory incident on the telescope assembly is assumed being omnidirectional, with the isotropic e- fluence coming from a $2 \pi$ steradians upon the telescope then onto the mirror. In order to test the consistency of our calculation we cross checked the results given by Spenvis (MULASSIS module) with the ones generated with our Geant4 based simulation software. In our computed approach the e- are fired from the surface of a fictitious sphere centered toward our optic (the radius of the sphere is assumed large compared to our telescope design in order to mimic at best the omnidirectional flux), see Figure 13.
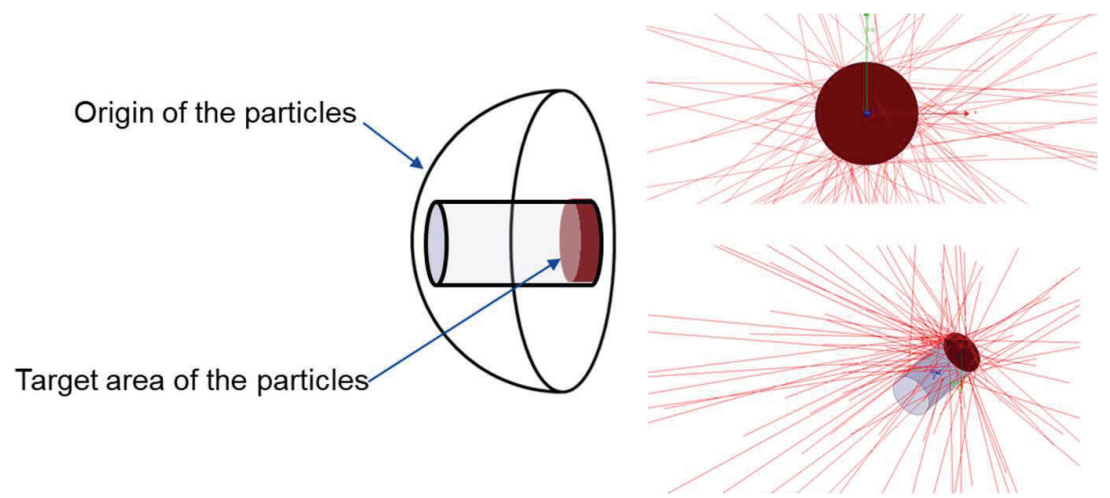

Figure 13: Left - In order to simulate a realistic space environment we assumed that e-particles were fired randomly from a virtual hemisphere centered on the simplified telescope geometry presented in Figure 12, the e- direction were oriented toward the optic in order to reproduce an omnidirectional particle flux. Contrarily to the image sketched on the right, the radius of the virtual sphere was

by far larger than the telescope. Right-typical trajectories of the particle fired onto the telescope model computed with Geant4.

Cross comparisons made with Spenvis on an unshielded system (i.e. without tube assembly) using the same electronic fluxes demonstrated consistency with the Geant4 simulations. See Figure 14 and Figure 15 for, respectively, for LEO and 
GEO orbits. The same simulations have been repeated taking into account this time the effect of the telescope tube assembly. In both cases, we observe a tube screening effect that reduces by $\sim 15$ to 20 times the magnitude of the dose deposited in the first millimeters of the mirror's facesheet ${ }^{1}$.
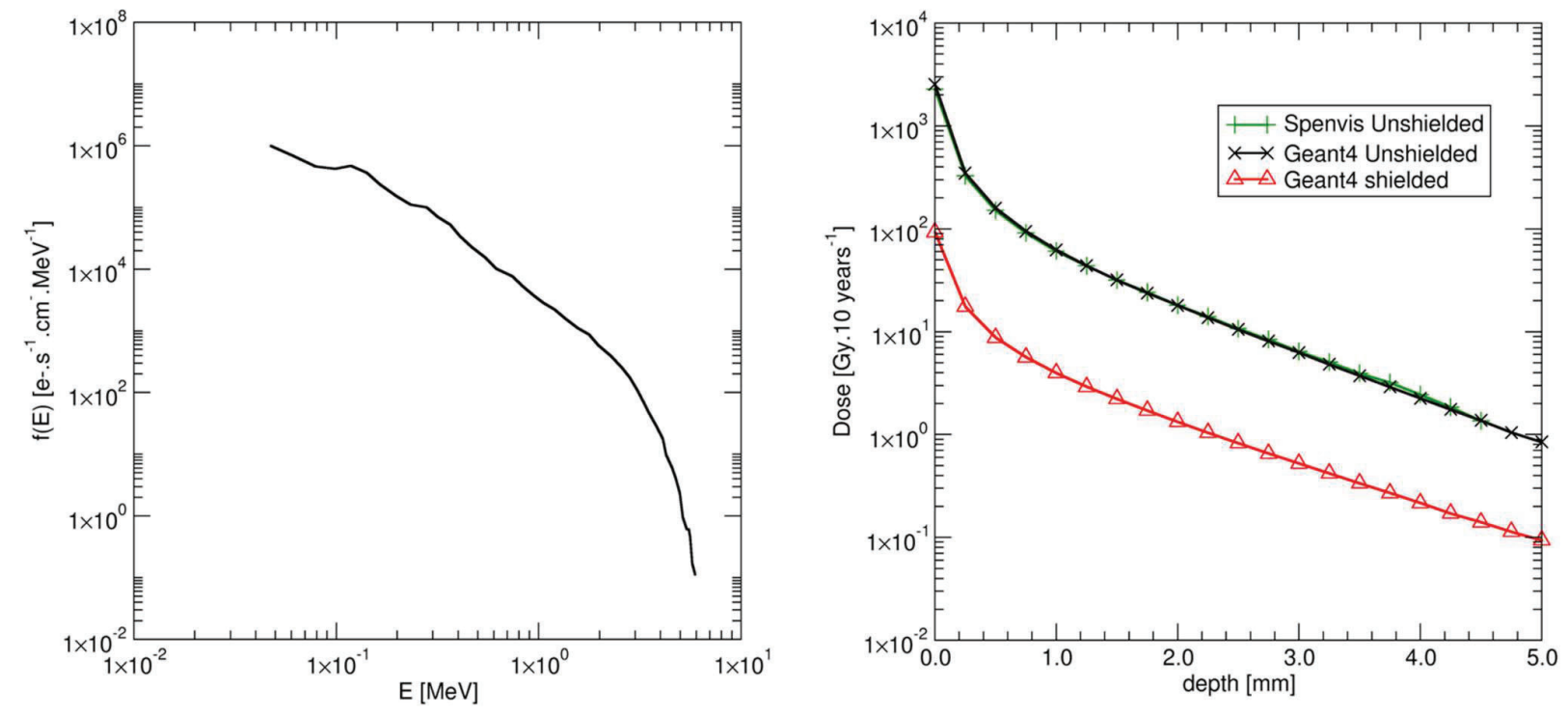

Figure 14: Left: energy distribution of e-calculated over a LEO orbit. Right: sagitta corresponding ZERODUR ${ }^{\circledR}$ dose-depth distribution calculated using Spenvis(+) and Geant4 (×) for a unshielded structure and for a shielded structure (4) over a 10 year long mission. N.b. the shielding proposed in this simulation is minimalist, as we do not take into account any structural and thermal shielding.

\footnotetext{
${ }^{1}$ The simple rescaling of the bare dose-depth profile by the solid angle measured from the center of the optic and delimited by the tube assembly is not satisfactory due to the presence of some multiple particle scattering along the baffle as well as bremsstrahlung effects.
} 

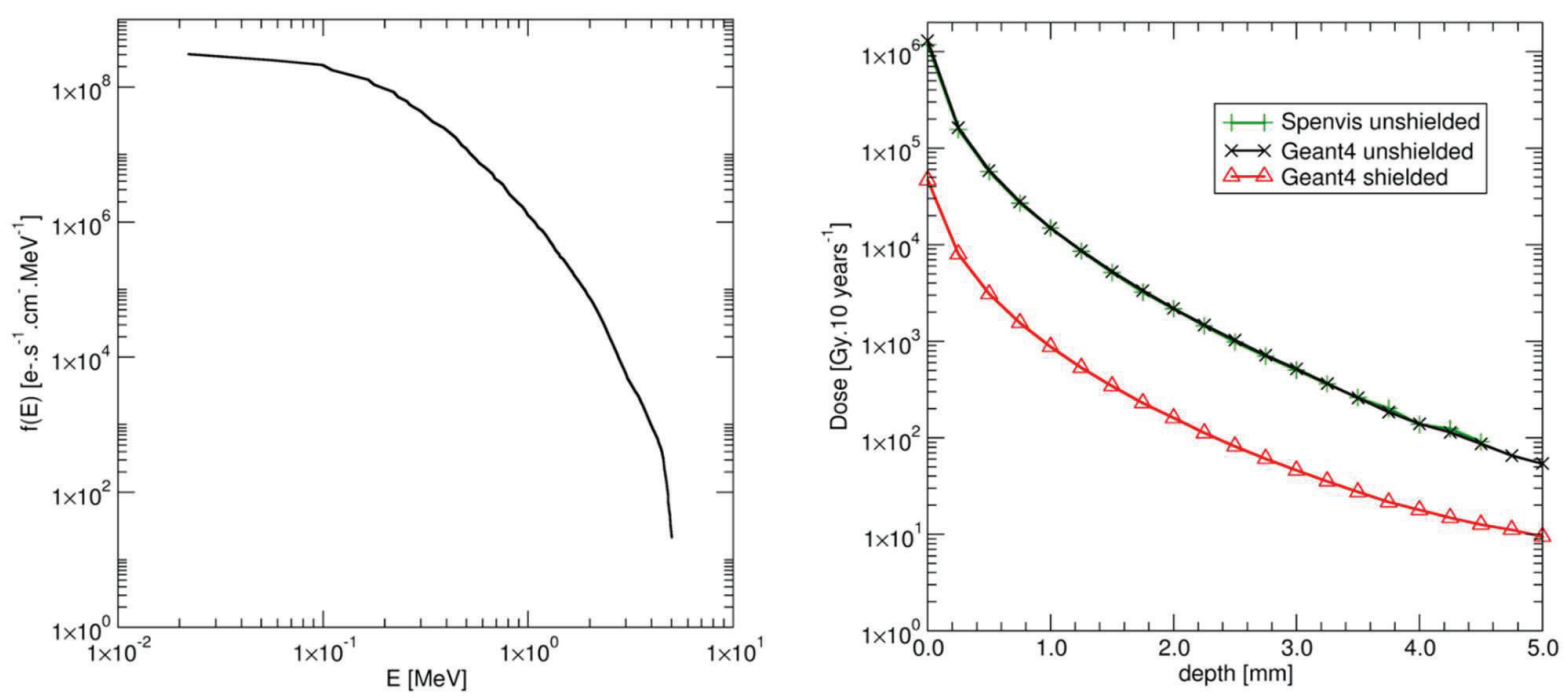

Figure 15: Left- energy distribution of e-calculated over a GEO orbit. Right-corresponding Zerodur ${ }^{\circledR}$ dose-depth distribution calculated using Spenvis(+) and Geant4 (×) for a unshielded structure and for a shielded structure (4) for a 10 year long mission. N.b. the shielding proposed in this simulation is minimalist, as we do not take into account any structural and thermal shielding.

The radial dose profile is also extremely instructive, see Figure 16, we can see these that the radiation profile is almost flat, which means that we are very close to the working hypothesis of the 1D continuous model presented at the very beginning of this paper, where the dose distribution only depends on the depth. The deformation expected for those radius independent profile are expected to have a pure power contribution. The power deformation can easily be compensated by refocusing the optic. 


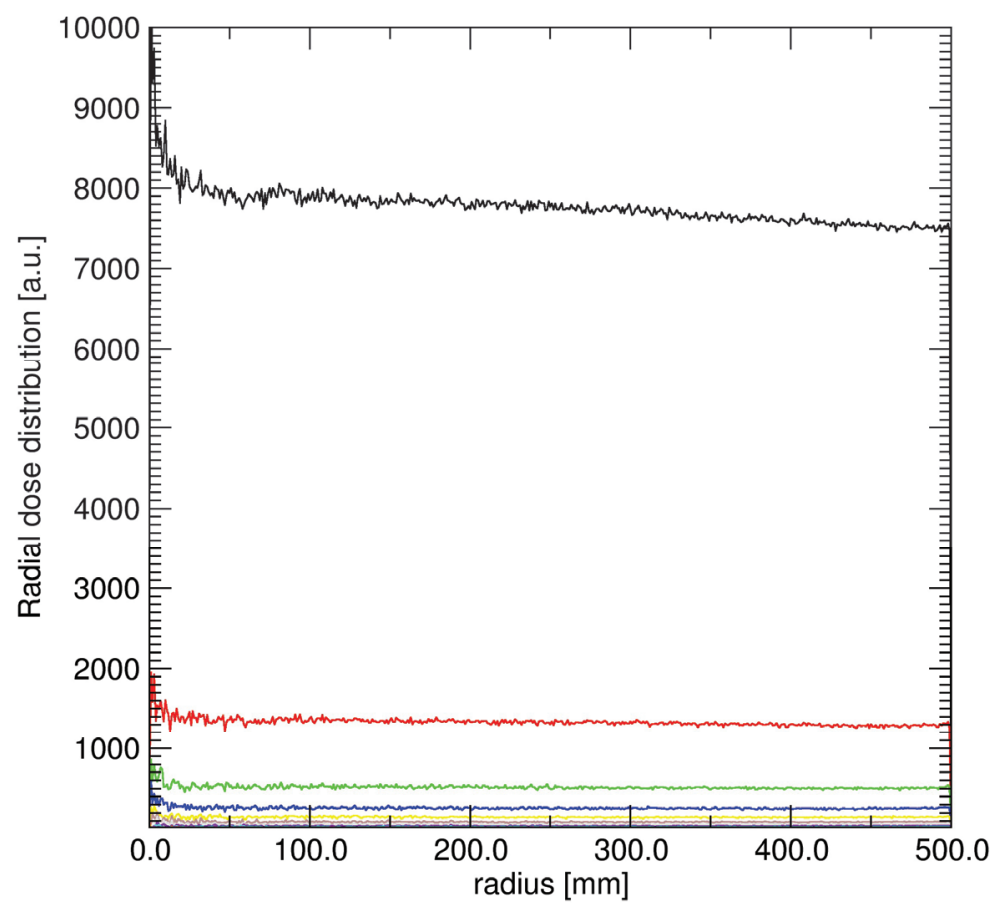

Figure 16: Radial distribution of the deposited dose calculated for the shielded geostationary orbit for different thicknesses (in different colors). The profile is extremely flat all over the radius and no edges effect are observed close to the interface ZERODUR ${ }^{\circledR}$ tubing. The small spikes close to $0 \mathrm{~mm}$ are a pure statistical artefact in this area.

\subsection{D estimated change of curvature}

The TID profile generated on the shielded system then have been used in the continuous 1D approach. We still considered a $\emptyset=1 \mathrm{~m}$ telescopes but because we are estimating bending, we take into account the isogrid structure of a $75 \mathrm{~mm}$ bulk mirror thickness (empirically found to be equivalent to $100 \mathrm{~mm}$ isogrid thickness). We first consider the telescope subject to a 10 years e- irradiation over two different orbits, namely LEO and GEO, with and without shielding. We next evaluate the effect of the TID on the local CTE and its consequence if the temperature of the mirror (isothermal assumption) is drifting by about $\Delta T=5 \mathrm{~K}$ from the nominal operating temperature $T_{o p}$ :

$$
\varepsilon(z) \propto \operatorname{CTE}\left(T_{o p,} ; D(z)\right)
$$

The results of this model are presented on the Figure 17 below: 


\begin{tabular}{|c|c|c|c|c|c|c|c|c|}
\hline \multirow[b]{3}{*}{$\mathrm{T}_{\text {op }}\left[{ }^{\circ} \mathrm{C}\right]$} & \multicolumn{4}{|c|}{ LEO } & \multicolumn{4}{|c|}{ GEO } \\
\hline & \multicolumn{2}{|c|}{ unshielded } & \multicolumn{2}{|c|}{ shielded } & \multicolumn{2}{|c|}{ unshielded } & \multicolumn{2}{|c|}{ shielded } \\
\hline & $\operatorname{RoC}[\mathrm{m}]$ & $\mathrm{PV}[\mathrm{nm}]$ & $\mathrm{RoC}[\mathrm{m}]$ & $\mathrm{PV}[\mathrm{nm}]$ & $\operatorname{RoC}[\mathrm{m}]$ & $\mathrm{PV}[\mathrm{nm}]$ & $\operatorname{RoC}[\mathrm{m}]$ & $\mathrm{PV}[\mathrm{nm}]$ \\
\hline-15 & $-2,76 E+08$ & $-0,45$ & \begin{tabular}{|l|}
$-9,02 E+08$ \\
\end{tabular} & $-0,14$ & $-2,49 \mathrm{E}+07$ & $-5,02$ & $-8,02 \mathrm{E}+07$ & $-1,56$ \\
\hline-10 & $-2,46 E+08$ & $-0,51$ & $-8,04 \mathrm{E}+08$ & $-0,16$ & $-2,22 \mathrm{E}+07$ & $-5,63$ & $-7,15 E+07$ & $-1,75$ \\
\hline-5 & $-2,32 E+08$ & $-0,54$ & $-7,25 E+08$ & $-0,17$ & $-2,00 E+07$ & $-6,24$ & $-6,45 E+07$ & $-1,94$ \\
\hline 0 & $-2,11 E+08$ & $-0,59$ & $-6,62 E+08$ & $-0,19$ & $-1,82 \mathrm{E}+07$ & $-6,85$ & $-5,87 E+07$ & $-2,13$ \\
\hline 5 & $-1,94 E+08$ & $-0,64$ & $-6,08 E+08$ & $-0,21$ & $-1,68 \mathrm{E}+07$ & $-7,46$ & $-5,39 \mathrm{E}+07$ & $-2,32$ \\
\hline 10 & $-1,79 \mathrm{E}+08$ & $-0,70$ & $-5,62 E+08$ & $-0,22$ & $-1,55 E+07$ & $-8,08$ & $-4,98 \mathrm{E}+07$ & $-2,51$ \\
\hline 15 & $-1,67 E+08$ & $-0,75$ & $-5,22 E+08$ & $-0,24$ & $-1,44 \mathrm{E}+07$ & $-8,70$ & $-4,63 E+07$ & $-2,70$ \\
\hline 20 & $-1,56 E+08$ & $-0,80$ & $-4,88 E+08$ & $-0,26$ & $-1,34 \mathrm{E}+07$ & $-9,31$ & $-4,33 E+07$ & $-2,89$ \\
\hline
\end{tabular}

Figure 17: Induced VRoC and in the PV surface for a LEO and GEO orbit assuming a shielded and an unshielded system for an overall temperature shift of about $\Delta T=5 \mathrm{~K}$ the operating temperature, the operating temperatures are reminded in the left column.

We can see that the impact of electrons is only marginal; moreover, one should keep in mind that these deformations are mostly defocus $^{2}$ and can be easily mechanically corrected. Practically the reflective optic is also bound to the satellite structure; these connecting points are increasing the stiffness of the overall optic thus reducing further the deformations.

In a second time, we showed the effect of a local temperature gradient of about $\delta T=0,75 \mathrm{~K}$ along the $\mathrm{d}=75 \mathrm{~mm}$ thickness of mirror onto the $\mathrm{VRoC}$ and $\mathrm{PV}$ (i.e $1 \mathrm{~K}$ over a $100 \mathrm{~mm}$ thick isogrid system), we performed these calculations taking also into account the change of CTE induced by the TID. In this configuration, the strain can be written:

$$
\varepsilon(z) \propto \operatorname{CTE}\left(T_{o p .} ; D(z)\right)\left(T_{o p .}+\frac{\delta T * z}{d}\right)
$$

The calculation have been performed assuming two different materials, on one hand CO720® Cordierite material from Kyocera (unirradiated) and on the other hand ZERODUR ${ }^{\circ}$ (unirradiated and irradiated assuming LEO/GEO orbits). The instant CTE of these two material are compared on Figure 18. The VRoC and the surface PV are provided on Figure 19.

\footnotetext{
${ }^{2}$ The shielding due to the baffle induces only a weak radial dependence of the dose-depth field. In addition to this, the optic is fixed to the satellite, strengthening thus the overall stiffness of the satellite.
} 


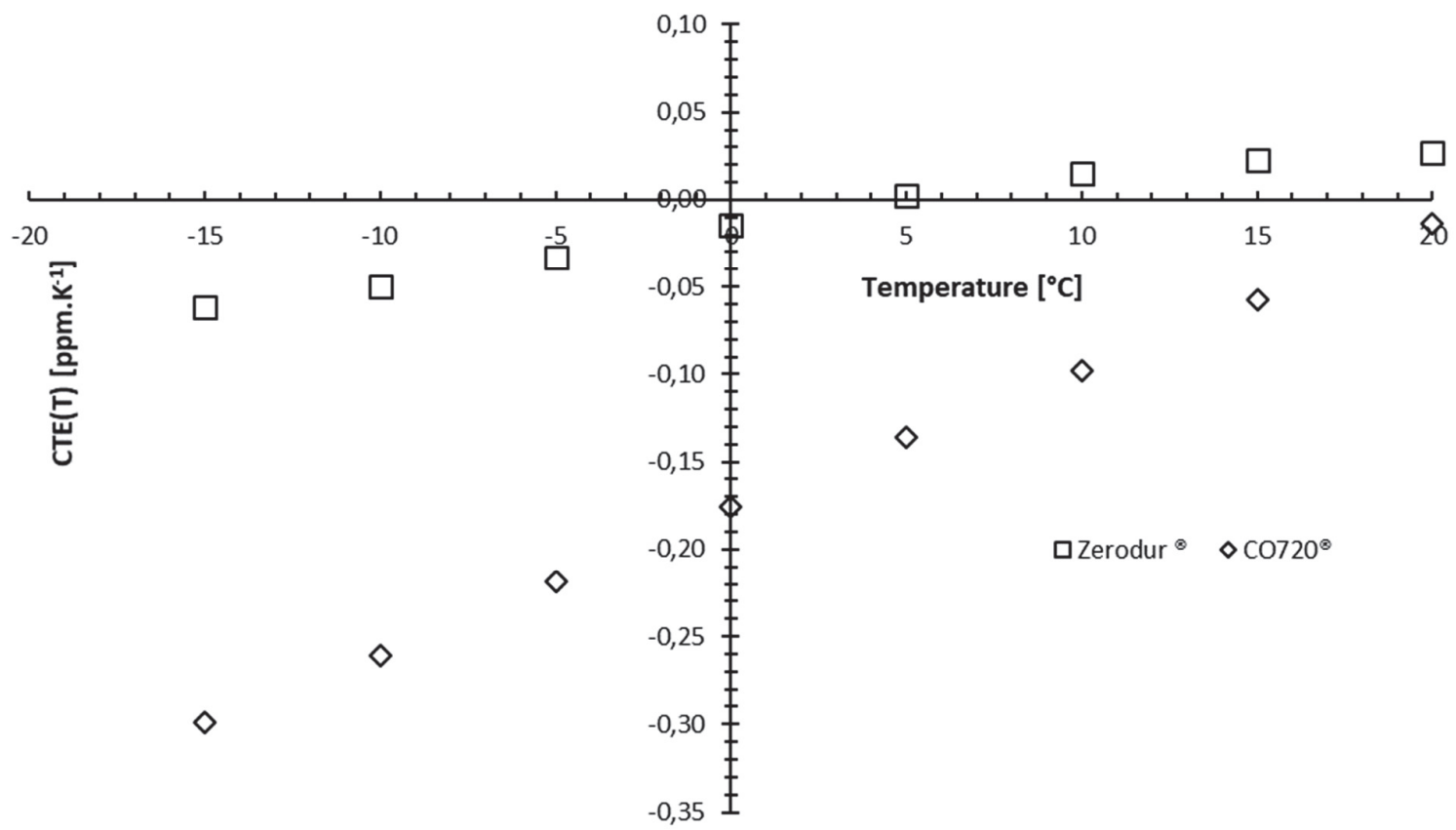

Figure 18: Comparison between the instant CTE(T) of ZERODUR ${ }^{\circledR}$ (squares) and CO720® (diamond) over a $[-15 ; 20]^{\circ} \mathrm{C}$ temperature range.

\begin{tabular}{|c|c|c|}
\cline { 2 - 3 } \multicolumn{1}{c|}{} & \multicolumn{2}{c|}{$\mathrm{CO} 20^{\otimes}$ without irr. } \\
\cline { 2 - 3 } \multicolumn{1}{c|}{} & \multicolumn{2}{c|}{ Only T gradient effect } \\
\hline $\mathrm{T}_{\text {op. }}\left[{ }^{\circ} \mathrm{C}\right]$ & RoC $[\mathrm{m}]$ & $\mathrm{PV}[\mathrm{nm}]$ \\
\hline-15 & $3,35 \mathrm{E}+05$ & 373,34 \\
\hline-10 & $3,83 \mathrm{E}+05$ & 326,24 \\
\hline-5 & $4,58 \mathrm{E}+05$ & 273,11 \\
\hline 0 & $6,05 \mathrm{E}+05$ & 206,70 \\
\hline 5 & $7,34 \mathrm{E}+05$ & 170,23 \\
\hline 10 & $1,02 \mathrm{E}+06$ & 122,80 \\
\hline 15 & $1,74 \mathrm{E}+06$ & 71,66 \\
\hline 20 & $7,39 \mathrm{E}+06$ & 16,91 \\
\hline
\end{tabular}

\begin{tabular}{|c|c|c|c|c|c|c|c|c|c|c|}
\hline \multirow[b]{4}{*}{$\mathrm{T}_{\mathrm{op}}\left[{ }^{\circ} \mathrm{C}\right]$} & \multirow{3}{*}{\multicolumn{2}{|c|}{$\begin{array}{l}\text { Zerodur }{ }^{\otimes} \text { without irr. } \\
\text { Only T gradient effect }\end{array}$}} & \multicolumn{8}{|c|}{ Zerodur $^{(6)}+$ irr. } \\
\hline & & & \multicolumn{4}{|c|}{ LEO } & \multicolumn{4}{|c|}{ GEO } \\
\hline & & & \multicolumn{2}{|c|}{ unshielded } & \multicolumn{2}{|c|}{ shielded } & \multicolumn{2}{|c|}{ unshielded } & \multicolumn{2}{|c|}{ shielded } \\
\hline & $\operatorname{RoC}[\mathrm{m}]$ & $\mathrm{PV}[\mathrm{nm}]$ & $\operatorname{RoC}[\mathrm{m}]$ & $\Delta P V[\mathrm{~nm}]$ & $\operatorname{Roc}[\mathrm{m}]$ & $\Delta P V[\mathrm{~nm}]$ & $\operatorname{RoC}[\mathrm{m}]$ & $\Delta \mathrm{PV}[\mathrm{nm}]$ & $\operatorname{Roc}[\mathrm{m}]$ & $\Delta \mathrm{PV}[\mathrm{nm}]$ \\
\hline-15 & $1,62 E+06$ & 77,35 & $-1,54 E+08$ & $-0,06$ & $-1,61 E+08$ & 0,02 & $-8,86 E+07$ & $-0,66$ & $-1,30 E+08$ & $-0,20$ \\
\hline-10 & $2,00 E+06$ & 62,35 & $-1,14 \mathrm{E}+08$ & $-0,06$ & $-1,19 \mathrm{E}+08$ & 0,02 & $-7,03 E+07$ & $-0,74$ & $-9,90 E+07$ & $-0,23$ \\
\hline-5 & $3,07 E+06$ & 40,72 & $-1,99 E+08$ & $-0,07$ & $-2,16 E+08$ & 0,02 & $-8,96 \mathrm{E}+07$ & $-0,84$ & $-1,54 E+08$ & $-0,26$ \\
\hline 0 & $7,04 \mathrm{E}+06$ & 17,74 & $-1,47 E+08$ & $-0,08$ & $-1,57 E+08$ & 0,03 & $-7,36 \mathrm{E}+07$ & $-0,93$ & $-1,19 E+08$ & $-0,28$ \\
\hline 5 & $-4,46 \mathrm{E}+07$ & $-2,80$ & $-1,08 E+08$ & $-0,09$ & $-1,14 \mathrm{E}+08$ & 0,03 & $-5,99 \mathrm{E}+07$ & $-1,02$ & $-9,04 E+07$ & $-0,31$ \\
\hline 10 & $-6,72 \mathrm{E}+06$ & $-18,61$ & $-8,38 \mathrm{E}+07$ & $-0,10$ & $-8,77 \mathrm{E}+07$ & 0,03 & $-4,99 \mathrm{E}+07$ & $-1,11$ & $-7,20 \mathrm{E}+07$ & $-0,34$ \\
\hline 15 & $-4,33 E+06$ & $-28,85$ & $-5,58 E+07$ & $-0,10$ & $-5,76 E+07$ & 0,03 & $-3,75 E+07$ & $-1,20$ & $-4,99 E+07$ & $-0,37$ \\
\hline 20 & $-3,66 E+06$ & $-34,17$ & $-4,88 E+07$ & $-0,11$ & $-5,03 E+07$ & 0,03 & $-3,34 \mathrm{E}+07$ & $-1,29$ & $-4,39 E+07$ & $-0,39$ \\
\hline
\end{tabular}

Figure 19: Change of PV calculated for a ZERODUR ${ }^{\circledR}$ mirror under a 0,75 K/75 mm (i.e. $1 \mathrm{~K} .100 \mathrm{~mm}-1$ ) temperature gradient around different operating temperatures $T_{\text {op. }}$. The variation of the CTE induced by the radiations have only a very marginal effect on the optical figure. The deformation are dominated by the instant CTE value.

These calculations show that a gradient of temperature through the thickness has a by far more significant effect than a homogeneous temperature drift within an irradiation aged material. In this situation, the effect of the irradiation is almost negligible. This effect has been graphically represented on Figure 20. 


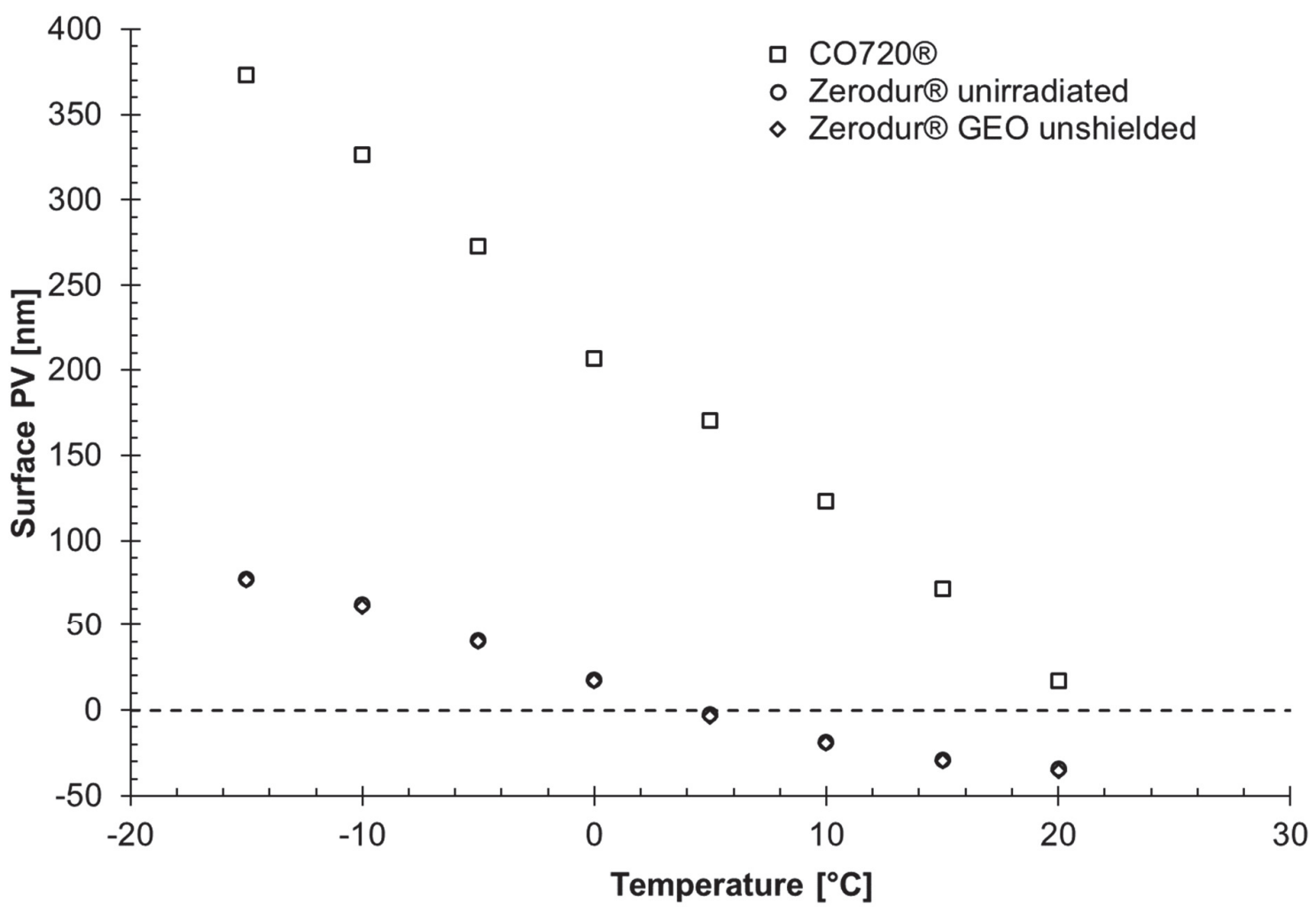

Figure 20: Surface PV in $\mathrm{nm}$ resulting from a 1,0 K.100 $\mathrm{mm}^{-1}$ temperature gradient for a $\varnothing=1 \mathrm{~m}$ mirror calculated for two different materials, square: $C O 720 ®$ and round/diamond Zerodur ${ }^{\circledR}$ respectively unirradiated and irradiated according to a GEO unshielded scenario.

We can see that the $\mathrm{CO} 720 \AA$ is as $\operatorname{good}$ a Zerodur ${ }^{\circledR}$ very close to it optimal operating temperature (around $23^{\circ} \mathrm{C}$ ), but small variations of the operating temperature from the CTE null-crossing point already imply significant thermal strain and consequently some significant changes in VRoC.

Practically, it is unusual and difficult to drive a telescope to isothermal as high as $T \approx 23{ }^{\circ} \mathrm{C}$. Generally, steady-state gradients are present and acceptable in the baffles and structures. More complex are asymmetric transients introduced when the pointing vector changes with respect to the sun, or in the case of a earth observing system, for which solar view factors modulate with orbital phase. Ideally, the primary mirror is maintained under stable thermal conditions, not undergoing time-varying gradients. With time-varying gradients, performance is affected whether the material is high diffusivity, high CTE or low diffusivity, low CTE. These efforts include insulation, and in extreme cases, proportional heat radiated on the mirror. If a telescope primary mirror is to operate as warm as Room Temperature $(\sim 23-\mathrm{C})$, heat must be radiatively added to the back of the mirror. If the front of the telescope is looking at cold space, the face of the mirror will want to cool as the mirror and baffles look at this cold space, thus a gradient is present, with the principal thermal resistance being the reflective coating of the mirror.

\section{CONCLUSION}

In this paper, we estimated the expected change of sagitta due to ionizing irradiation effect onto the CTE of Zerodur ${ }^{\circledR}$. We first determined at the lab scale the impact of electrons onto the CTE for different TID in order to propose a phenomenological law. We then investigated the expected irradiation profile within our material over a LEO and a GEO orbit without and with baffle shielding. We then combined these dose-depth scenarios with our CTE ageing law in order to investigate by means of a continuous plate theory two scenario. In the first scenario we considered a worn Zerodur ${ }^{\circledR}$ facesheet under a spatially homogeneous temperature drift, the observed cambra is then due to the local change in the CTE 
caused by the irradiation field. In this approach, the change in surface PV is only very marginal. In the second scenario we introduced some more complexity by adding a thermal gradient through the thickness of the Zerodur ${ }^{\circledR}$ facesheet. These calculations showed once more that the effect of irradiation are only contributing marginally to the change observed in the VRoC.

These calculations are all based on a 1D model whose validity has been previously cross-checked with FEM. However these results are assuming a free-free boundary conditions and are not taking into account any radial temperature distribution nor any complexity due to the isogrid structure of the reflective optic. In addition to this the CTE ageing law proposed in this work has been derived with a small dataset, the CTE being only marginally impacted by the ionization we only had two set of linear independent results which were significantly drifting from the baseline for feeding the fitting procedure. The generation of another set of data for a deposited dose of e.g. about $10^{7} \mathrm{~Gy}$ would significantly help us in confirming the power law ansatz used for the description of the CTE changes. All these aforementioned aspects must be and will be further deepened in some future contributions.

\section{REFERENCES}

[1] Döhring T., Hartmann, P., Lentes, F-T, Jedamzik, R, Davis M. J., Proc. SPIE (7425), 74250L (2009).

[2] Pepi, J. W., "Opto-structural Analysis", SPIE Press Bellingham, Washington (2018).

[3] Vasudevan, M., Johnson, W., Appl. Sci. Res. B, (9), 420-430 (1961).

[4] Carré, A., Hull, T., Valente, M., Krieg, J., Westerhoff, T., ICSO 2020 Paper session 249.

[5] Allison, J. et al, Nuclear Instruments and Methods in Physics Research Section A: Accelerators, Spectrometers, Detectors and Associated Equipment, (835), 186-225 (2016).

[6] Higby, P. L., Friebele, E. J., Shaw, C. M., Rajaram M., Graham, E. K., Kinser, D. L., Wolff, E. G., J. Am. Ceram. Soc. (71), 796-802 (1988).

[7] Bourrieau, J., Roméro, M., ESA Spacecraft Mater. In Space Environment, 275-285 (1979).

[8] Bourrieau, J., et al, « Étude expérimentale sur l'irradiation par les particules à haute energie des miroirs utilisés dans le télescope radiomètre METEOSAT », Report N²145/74/CG (1974).

[9] Seltzer, S., „SHIELDOSE: A Computer Code for Spahce-Shielding Radiation Dose Calculations“, National Bureau of Standards Technical Note 1116, U.S. Gov. Printing Office 1980.

[10] SPENVIS - "Space Environment Information System", https://www.spenvis.oma.be/credits.php hosted by the Belgian Federal Science Policy Office. 